\title{
Correction to: A stress-responsive transcription factor PeNAC1 regulating beta-D-glucan biosynthetic genes enhances salt tolerance in oat
}

\author{
Xiao-Dong Liang ${ }^{1,2} \cdot$ Mohamed Shalapy $^{2} \cdot$ Shi-Feng Zhao ${ }^{3} \cdot$ Jing-Hui Liu $^{4} \cdot J^{\text {Jun-Ying Wang }}{ }^{2}$ (I)
}

Published online: 7 December 2021

(c) Springer-Verlag GmbH Germany, part of Springer Nature 2021

\section{Correction to: Planta (2021) 254:130 \\ https://doi.org/10.1007/s00425-021-03770-6}

In this article the affiliation details for Mohamed Shalapy and Jun-Ying Wang were incorrectly given as XinJiang Academy of Agricultural Sciences Grain Crops Institute, No. 403 Nanchang Road, Urumqi 830091, China but should have been Biotechnology Research Institute of Chinese Academy of Agricultural Sciences, No. 12 Zhong Guan Cun South Street, Beijing 100081, China.

Publisher's Note Springer Nature remains neutral with regard to jurisdictional claims in published maps and institutional affiliations.

The original article can be found online at https://doi.org/10.1007/ s00425-021-03770-6.

\section{Jing-Hui Liu}

cauljh@163.com

$\bowtie$ Jun-Ying Wang

wangjunying@caas.cn

1 Present Address: XinJiang Academy of Agricultural Sciences Grain Crops Institute, No. 403 Nanchang Road, Urumqi 830091, China

2 Biotechnology Research Institute of Chinese Academy of Agricultural Sciences, No. 12 Zhong Guan Cun South Street, Beijing 100081, China

3 Zhangjiakou Academy of Agricultural Sciences, Zhangjiakou 075000, China

4 Inner Mongolia Agriculture University, No. 275 Xue Yuan East Street, Hohhot 010019, China 